\title{
Tau lepton reconstruction and identification with the ATLAS detector at the LHC
}

\author{
Aldo F. Saavedra*, on behalf of the ATLAS collaboration. \\ The University of Sydney \\ E-mail: a.saavedra@physics.usyd.edu.au
}

\begin{abstract}
The overall performance of the idenfication and measurements of hadronically decaying $\tau$ leptons in a wide dynamic range of transverse energies, spanning from (10-15) $\mathrm{GeV}$ up to at least $500 \mathrm{GeV}$, with the ATLAS detector at the LHC is presented. Hadronically decaying $\tau$ leptons are reconstructed by matching narrow calorimetric clusters with a small number of tracks. Two complementary approaches, the calorimeter based and the track based algorithm, have been developed to efficiently reconstruct these decays while providing the required large rejection rates against otherwise overwhelming backgrounds from jet production via QCD processes. Specific performance aspects of particular interest for the reconstruction of hadronic $\tau$ candidates are discussed together with the overall performance in terms of efficiency versus rejection rate of the large background from QCD jets expected at the LHC. Prospects for measurements with first data such as the determination of fake rates are discussed.
\end{abstract}

Prospects for Charged Higgs Discovery at Colliders

16-19 September 2008

Uppsala, Sweden

*Speaker. 


\section{Introduction}

The main aim of the new proton-proton collider, located at the European Particle Physics Laboratory (CERN) in Switzerland, is to discover the physics that will either confirm the Standard Model or provide a hint of new physics. The collider will be operated at a centre of mass energy of up to $14 \mathrm{TeV}$ with a design luminosity of $10^{34} \mathrm{~cm}^{-2} \mathrm{~s}^{-1}$. Tau leptons in the final state are important signatures for physics beyond the Standard Model like supersymmetric models (SUSY).

The $\tau$ lepton decays into a $\tau$ neutrino and either leptons or hadrons. The branching fraction of the decay is about 35\% into leptons and 65\% into hadrons[1]. This can be categorised into decays with one charged hadron (one prong) and multiple charged hadrons ( 3 prong) with relative branching fractions of $77 \%$ and $23 \%$, respectively. The ATLAS experiment is sensitive to both kinds of decays in the transverse momentum $\left(p_{\mathrm{T}}\right)$ range between $10 \mathrm{GeV}$ and $500 \mathrm{GeV}$. This report will focus on the reconstruction and identification of the hadronically decaying $\tau$ leptons.

\section{The ATLAS Detector}

The ATLAS detector[2] is one of three detectors designed and built to study the proton-proton collisions at the LHC. Its design has been optimised to discover and to study a wide range of processes and proposed theoretical models.

The sub-detectors that play main roles in the reconstruction of hadronically decaying $\tau$ leptons are the Inner Detector (ID) and the calorimeter system. The Inner Detector is a standalone tracking detector located within a solenoid which produces an axial magnetic field of $2 \mathrm{~T}$. It consists in the innermost detector region of three layers of Silicon Pixel modules, followed by four layers of Silicon strip modules and complemented by a Transition Radiation Tracker (TRT) composed of polyimide drift tubes. This provides a complete coverage in the azimuthal coordinate $(\phi)$ and $|\eta|<2.5$ in pseudorapidity. From the hits registered by the ID the trajectory, momentum and charge of the particles can be determined. The high precision measurements with the silicon detectors allow for an accurate impact parameter determination and vertex reconstruction while a robust pattern recognition is achieved by having the precision trackers at small radii and the TRT at a larger radius.

To determine the expected tracking performance for primary tracks Monte Carlo studies were performed for a range of different particle types and momenta [3]. The efficiency of the pion reconstruction and resolution of the track parameters, such as the impact parameter, is shown in Fig. 1. The efficiency for pions is affected by multiple scattering and hadronic interactions in the material of the ID. The left graph of Fig. 1 shows how the drop in efficiency follows the increase in accumulated material inside the ID as a function of $\eta$. The right graph of Fig. 1 shows how the resolution of the measured impact parameter is also affected by this.

The second ingredient is the energy measured by the calorimeter system. The ATLAS calorimeter system consists of a number of sampling calorimeters providing full $(\phi)$ coverage and up to $|\eta|<3.2$. The electromagnetic (EM) calorimeters are implemented as lead/liquid argon detectors with its absorbers and electrodes possessing an accordion geometry operated at a nominal voltage of $2000 \mathrm{~V}$ to collect the signal. The barrel $(0<|\eta|<1.35)$ and the end cap $(1.5<|\eta|<2.5)$ possess three precision layers of different granularity. In the region where the coverage between 

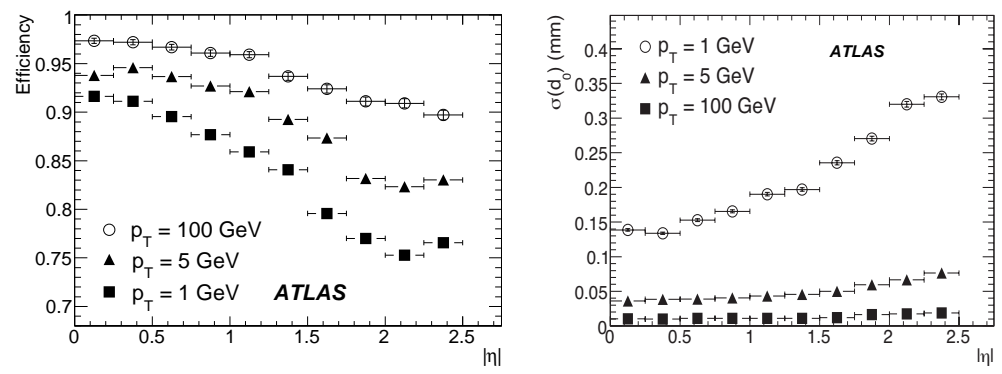

Figure 1: The efficiency(left) and transverse impact parameter resolution(right) for monte carlo pion tracks $\left(p_{\mathrm{T}} 1 \mathrm{GeV}, 10 \mathrm{GeV}\right.$ and $100 \mathrm{GeV}$ ) as a function of $\eta$.

them overlaps $(1.35<|\eta|<1.5)$ the barrel and end cap have two precision layers. Only a small fraction of pions are expected to lose a substantial fraction of their total energy (3\% of $10 \mathrm{GeV}$ pions deposit more than $8 \mathrm{GeV}$ ) because the calorimeter radial depth is only about one interaction length $(\lambda)$.

The hadronic calorimeter, located behind the EM calorimeters, is implemented using two technologies. The central region $(0<|\eta|<1.7)$ has a radial length of $7.4 \lambda$ where the absorber is steel and scintillator tiles are the sampling material. The tiles are oriented radially and normal to the beams. The end caps $(1.7<|\eta|<3.2)$ are instrumented using copper/liquid argon with the wheels sharing the same cryostat as the EM end caps and Forward Calorimeter. The central region has three layers while the end cap possesses four.

To determine the performance of the tracking system and calorimeters, a number of testbeam runs with single energy beams were conducted at CERN[2]. The results are shown in Fig. 2. The first graph shows the fractional momentum resolution $\left(\sigma_{p} / p\right)$ as a function of the pion momentum after alignment was performed. This is in agreement with the results from Monte Carlo studies of the system with perfect alignment. In the case of the EM calorimeter the reconstructed energy response was measured to be linear within $\pm 0.1 \%$ for the range $15-180 \mathrm{GeV}$ and within $\pm 0.2 \%$ for the hadronic calorimeter. The last two graphs of figure 2 feature the fractional energy resolution $\left(\sigma_{E} / E\right)$ as a function of beam energy for the calorimeter modules tested. The results of the EM calorimeter for electrons are shown in graph 2 while the third graph provides the results of the combined calorimeter system (the EM and hadronic calorimeters) as a function of the inverse square root of the energy of the incident pions. The testbeam data for these two graphs was fitted with the function $\frac{\sigma(E)}{E}=\frac{a}{\sqrt{E(G e V)}} \oplus b$ where $a$ is the stochastic term (or sampling term) and $b$ the constant term reflecting local non-uniformities in the response of the calorimeter, with addition of the two terms being done in quadrature. The fitted values are shown on the graphs in Fig. 2, where the quoted errors are statistical only.

The following sections explains how the measurements performed by the ID and calorimeter system are used to create a reconstructed $\tau$ candidate.

\section{Hadronic Tau Reconstruction}

Tau reconstruction requires the identification of a set of good quality tracks with low multi- 

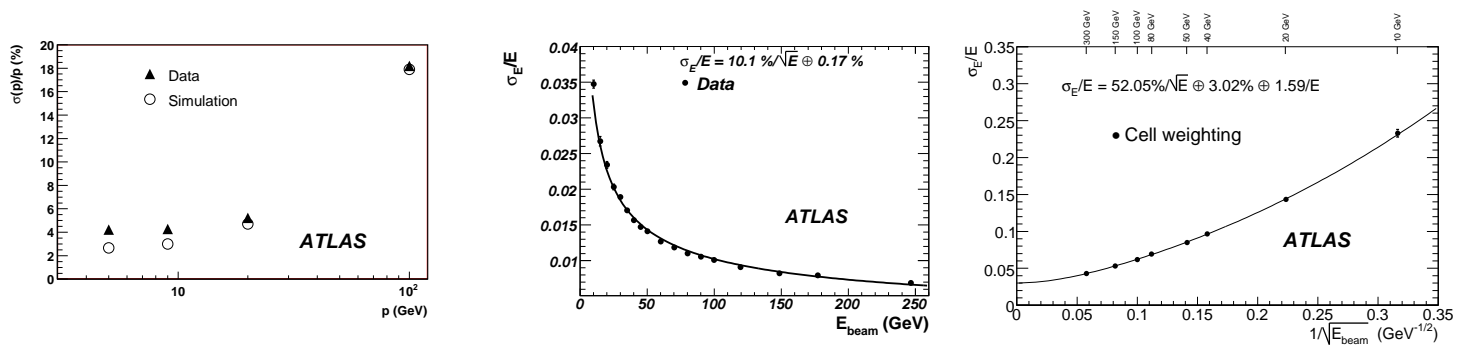

Figure 2: Results from the Combined Testbeam[2]. The first graph on the left features $\sigma_{p} / p$ of the ID for pions with respect to their momentum. Next, $\sigma_{E} / E$ of the EM calorimeter is shown as a function of beam energy for electrons. The last graph shows $\sigma_{E} / E$ for pions as a function of the inverse square root of the beam energy for the EM and hadronic calorimeters.

plicity which are well collimated in $(\eta, \phi)$. These tracks should not match any tracks in the muon spectrometer or be consistent with an electron (i.e. tracks should not possess hard bremsstrahlung or contain high threshold hits in the TRT). The calorimeter will measure the visible energy of the decay which excludes the neutrinos. The collimated decay products will create a relatively narrow shower in the EM calorimeter with a significant pure electromagnetic component. For example on average 55\% of the energy is carried by neutral pions in the single prong decay with one or more $\pi^{0} \mathrm{~s}$. Like the tracks, the resulting calorimeter clusters should be isolated from the rest of the event and close to the impact point of the tracks at the calorimeter face. There are two approaches to $\tau$ reconstruction and identification which have been developed[4]:

- Calorimeter based algorithm: The seed for the reconstructed object is based on clusters in the hadronic and EM calorimeters. Only clusters with transverse energies $\left(E_{\mathrm{T}}\right)>15 \mathrm{GeV}$ are considered. These are constructed from cells that are within a radius $(\Delta R)$ of less than 0.4 (which is the distance in the pseudorapidity-azimuthal angle space defined by $\Delta R=\sqrt{\Delta \eta^{2}+\Delta \phi^{2}}$ ) from the barycentre of the cluster and calibrated using an H1-style calibration[4]. Any track with $p_{\mathrm{T}}>2 \mathrm{GeV}$ that is within $\Delta R<0.3$ will be associated with the object. These candidates are referred to as Calo seeded $\tau$.

- Track based algorithm: This algorithm is seeded by tracks with a transverse momentum $\left(p_{\mathrm{T}}>6 \mathrm{GeV}\right)$ which is expected to reproduce to a certain degree the direction of the visible decay products because the tracks are well collimated. A number of good quality tracks found within the core region (a cone of $\Delta R<0.2$ whose axis is the direction of the leading track) and minimal energy deposit in the isolation region (an annulus around the candidate of $0.3<\Delta R<0.2$ ) is required for a candidate to be accepted as a $\tau$ candidate. The energy scale for this type of candidate is determined using the Energy Flow algorithm which will be presented later. These candidates are referred to as Track seeded $\tau$.

To determine the performance of each algorithm, measurements were performed on a number of signal and background Monte Carlo samples. Some results are presented for both next.

\subsection{Calorimeter Based Tau Reconstruction}

The reconstruction efficiency obtained from Monte Carlo studies reaches a plateau of $98 \%$ 

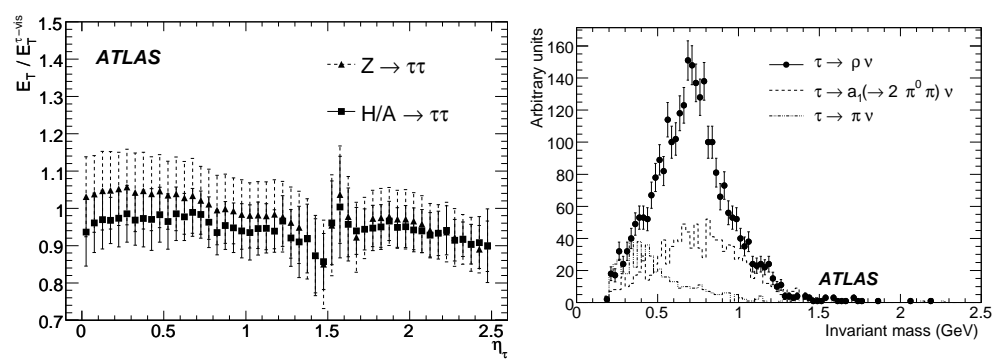

Figure 3: The left graph shows the ratio of the reconstructed $E_{\mathrm{T}}$ and Monte Carlo $E_{\mathrm{T}}^{\tau v i s}$ for calo-seeded $\tau$ candidates. The right graph presents the invariant mass distribution of the visible $\tau$ decay products obtained from different decay modes that included at least one reconstructed $\pi^{0}$ for track-seeded $\tau$ candidates.

for $\tau$ leptons with a transverse energy of their visible components $\left(E_{\mathrm{T}}^{\tau \mathrm{vis}}\right)$ greater than $30 \mathrm{GeV}$. The efficiency at the lower limit of $15 \mathrm{GeV}$ is $20 \%$ and the energy calibration of the clusters results in a $10 \%$ energy resolution. The left graph in Fig. 3 shows the mean ratio between the reconstructed $E_{\mathrm{T}}$ and Monte Carlo $E_{\mathrm{T}}^{\tau v i s}$ for $\tau$ candidates from two samples: $Z \rightarrow \tau \tau$ (squares) and $b \bar{b} A^{0} / H^{0}\left(m_{H}=800 \mathrm{GeV}\right) \rightarrow \tau \tau$ (triangles), as a function of $\eta$. The error bars represent the width of the Gaussian used to fit the distribution at each $\eta$ bin. The discontinuity can be attributed to the transition between the barrel and end cap region; the material distribution peaks in its vicinity due to the presence of the services.

\subsection{Track Based Tau Reconstruction}

The efficiency of the track based algorithm for $\tau$ leptons whose decay products include one charged hadron ( 1 prong) is around $80 \%$. For the case of three charged hadrons ( 3 prong) the efficiency is lower (50\%). The values were obtained by including only Monte Carlo $\tau$ candidates whose leading charged track has a $p_{\mathrm{T}}>9 \mathrm{GeV}$. The energy scale of the $\tau$ candidates was determined using the Energy Flow algorithm where the calorimeter energy from charged particles is replaced by the track(s) momenta. The contribution due to neutral pions is included by looking for EM clusters isolated from good quality tracks and looking for cells not used within $\Delta R=0.2$ of the impact point of the track at each layer of the EM calorimeter. The algorithm achieved a resolution of $8.6 \%$. The ability to differentiate between the deposited energy from neutral and charged particles allows the number of $\pi^{0} \mathrm{~s}$ in the decay to be determined. The graph on the right hand side of Fig. 3 shows the distribution of the invariant mass of the reconstructed visible decay products for different decay modes. Only single prong $\tau$ decays with at least one $\pi^{0}$ reconstructed are included.

\section{Tau Identification}

A number of quantities are associated with each reconstruction algorithm, whose purpose is to discriminate between the hadronic $\tau$ decays and fake candidates. These quantities have been defined to take advantage of the $\tau$ lepton kinematics and properties which result in distributions with different shapes for signal and background. Each algorithm possesses a number of these quantities which are used as inputs to its identification method. For the calorimeter based reconstruction a 

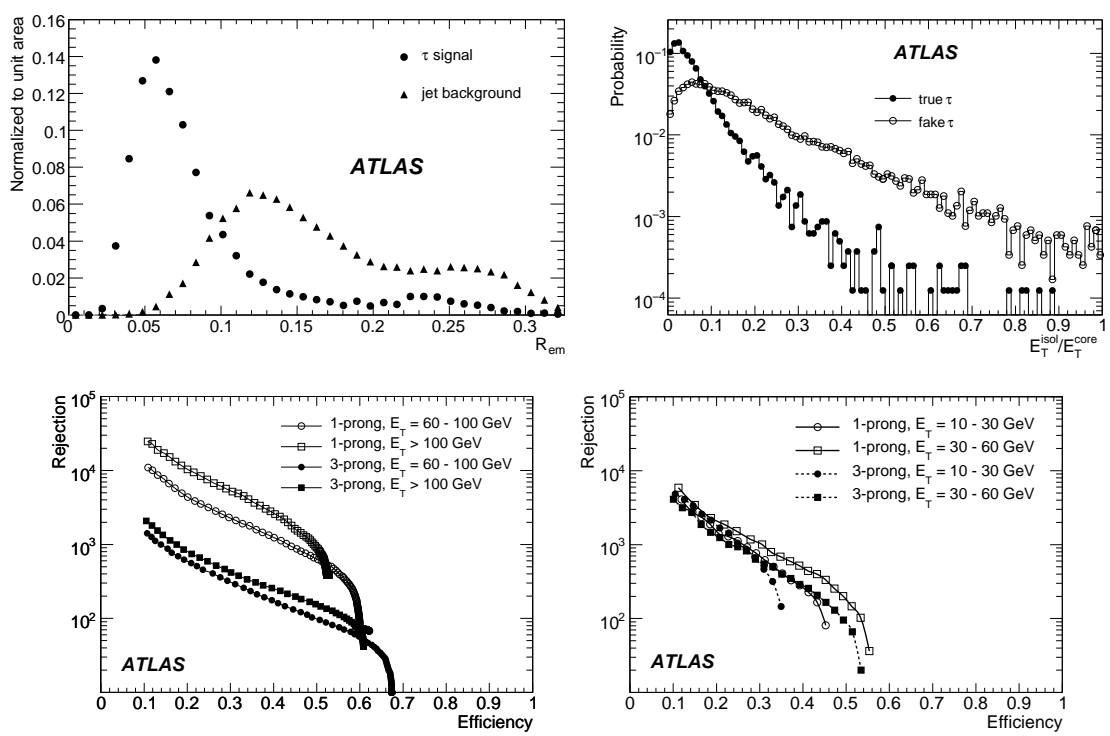

Figure 4: The top two graphs show an example of quantities calculated by each $\tau$ reconstruction algorithm (left calorimeter based and right track based) used to discriminate between $\tau_{\text {had }}$ decays and fakes due to QCD jets. The two bottom graphs show the determined rejection rate vs. efficiency for the respective identification algorithms whose inputs are the discriminating quantities. Likelihood function for calorimeter based on the left and neural network for track based on the right. See text for details.

likelihood function has been developed while a trained neural network is employed by the track based reconstruction. In Fig. 4, the distributions for a variable from each algorithm is shown as an example. On the top left the distribution of the $E_{\mathrm{T}}$ weighted by the distance between each cell and the barycentre of the cluster, $R_{e m}$, calculated by the calorimeter based algorithm is shown. The formula is $R_{e m}=\frac{\sum_{i=1}^{n} E_{\mathrm{T}, \mathrm{i}} \sqrt{\left(\eta_{i}-\eta_{\mathrm{cluster}}\right)^{2}+\left(\phi_{i}-\phi_{\mathrm{cluster}}\right)^{2}}}{\sum_{i=1}^{n} E_{\mathrm{T}, \mathrm{i}}}$ where $E_{\mathrm{T}, \mathrm{i}}$ is the transverse energy of the cell $i$ whose position is $\left(\eta_{i}, \phi_{i}\right)$. The top right graph is the ratio of $E_{\mathrm{T}}$ in the isolation and core region as calculated by the track based algorithm. Both are sensitive to the transverse shower profile which is smaller in hadronic $\tau$ decays. This is the reason why the distributions from signal peaks at lower values than the distribution from fakes due to QCD jets.

The expected performance of the identification algorithms is shown in the bottom graphs of Fig. 4. In each graph the rejection rate for Monte Carlo QCD dijets is plotted with respect to the selection efficiency of hadronically decaying $\tau$ leptons. The curves correspond to 1 prong and 3 prong candidates of different $p_{\mathrm{T}}$ ranges. For an identification efficiency of $30 \%$ the rejection rate is higher for 1 prong candidates than for 3 prong candidates. For 1 prong candidates the rejection rate increases with $p_{\mathrm{T}}$ from 740 to 1030 for track-seeded and $1130 \pm 50$ to $4370 \pm 280$ for calo-seeded $\tau$ candidates. The rejection rate is constant for 3 prong track-seeded candidates (590 \pm 70$)$ and increases with $p_{\mathrm{T}}$ from $187 \pm 3$ to $423 \pm 8$ for calo-seeded candidates.

\section{First Data}

One of the early measurements to be done with first data is to determine the fake rates and tune 

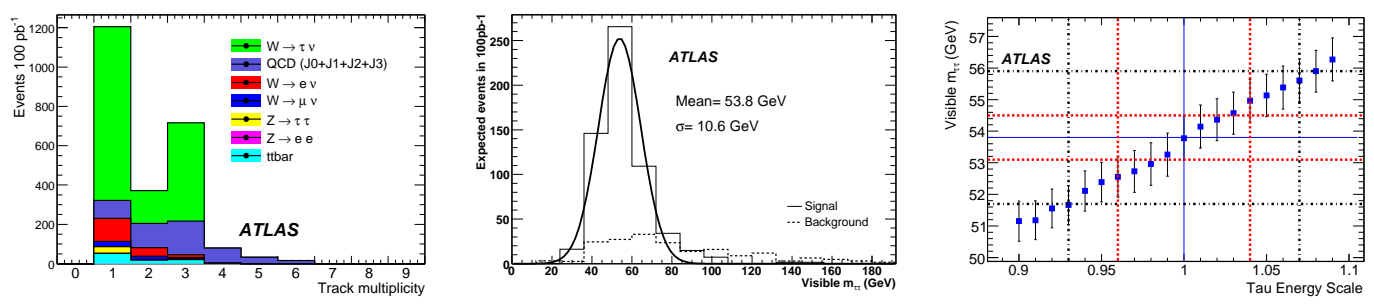

Figure 5: Expected results from first data studies $\left(100 \mathrm{pb}^{-1}\right)$. The first graph shows the track multiplicity for $\tau$ lepton candidates selected as described in the text. The next two graphs show the reconstructed visible mass of the $Z$ boson from a pair of lepton and $\tau_{\text {had }}$ and the visible mass as a function of $\tau_{\text {had }}$ energy scale.

the identification algorithms. This is necessary because the rejection rate for low $p_{\mathrm{T}} \tau$ leptons is of the order of $10^{-3}$ and the expected rate of QCD jets is much higher. This will be achieved with a clean QCD dijet sample collected by selecting two jets with similar $p_{\mathrm{T}}$ that are back to back. The other goal is to obtain a sample of $\tau$ leptons of high purity. With an integrated luminosity of 100 $\mathrm{pb}^{-1}$ from running at an instantaneous luminosity of $10^{31} \mathrm{~cm}^{-2} \mathrm{~s}^{-1}$ it is expected that a sample, of the order of thousands, of hadronically decaying $\tau$ leptons will be identified from the decays of $W$, $Z$ bosons and top quark pairs. This will allow the simulation and identification to be tuned. For example in Fig. 5 the first graph shows the track multiplicity of the $\tau$ candidates as contributing to the different processes. Taus with an $E_{\mathrm{T}}$ range of $20-60 \mathrm{GeV}$ were chosen and selected with an efficiency of $30 \%$. The QCD background was largely suppressed by placing a $60 \mathrm{GeV}$ cut on the missing energy of each event. The next two graphs are related to a study performed, using $Z \rightarrow \tau \tau \rightarrow \ell \nu \nu \tau_{\text {had }} \nu$ events, to determine the $\tau_{\text {had }}$ energy scale from the reconstructed $\ell \tau_{\text {had }}$ visible mass.

\section{Conclusion}

The ATLAS detector is designed to measure the visible decay products of the hadronically decaying $\tau$ leptons that will be produced in proton-proton collisions at the LHC. Two complementary reconstruction algorithms have been developed. Both offer identification procedures with high rejection rates for fake $\tau$ leptons from QCD jets for 1 prong and 3 prong $\tau$ candidates. Studies performed with the first $100 \mathrm{pb}^{-1}$ of data will enable the identification algorithms and simulation to be tuned allowing $\tau$ leptons to be a well understood probe for the search of new physics.

\section{References}

[1] C. Amsler et al. (Particle Data Group), Physics Letters B667, 1 (2008)

[2] The ATLAS Collaboration, The ATLAS Experiment at the CERN Large Hadron Collider, JINST 3, 2008 S08003.

[3] Cornelissen T, Elsing M, Gavrilenko I, Liebig W and Salzburger A, Single Track Performance of the Inner Detector New Track Reconstruction (NEWT), ATL-INDET-PUB-2008-002, March 2008.

[4] The ATLAS Collaboration, Expected Performance of the ATLAS Experiment, Detector, Trigger and Physics, CERN-OPEN-2008-020, Geneva 2008. 